Die Rolle der Maker-Community für eine nachhaltige Wirtschaft

\section{Fablabs und Hackerspaces}

Maker-Communities sind der soziale Resonanzraum, in dem erprobt, repariert, gedruckt und gehackt wird. Können daraus nachhaltige Wirtschaftsprozesse entstehen? Nein sagen einige, denn ein Teil der Maker haben nur Spaß daran, 3-D-Drucker mit Daten zu füttern, egal was raus kommt. Doch wenn man die wiederentdeckten zeitlichen und sozialen Produktionslogiken zu honorieren weiß, ist die Rolle der Maker nicht zu unterschätzen. Von Bastian Lange

$\mathrm{H}$ urra, wir haben ein neues marktfähiges Role Model: Der Maker. Friebe und Ramge haben es mit dem Slogan „Marke Eigenbau“ 2008 angedeutet. Er bezog sich auf die technologiebedingten Möglichkeiten von 3-D-Lasercutter und 3-D-Drucker, spezielle und wenige Gegenstände selbstständig zu entwerfen und zu bauen. Die Digitalisierung war der Garant der Distribution. Webplattformen wie Dawanda und Etsy ermöglichten den Sprung aus zu engen lokal-regionalen Kundenkreisen hin zu globalen Spezialisten- und Lieberhaberkäufern.

Seit zwei bis drei Jahren gesellen sich zu der Praxis des Selbermachens die dafür nötigen Gemeinschaftsräume. Diese Werkstätten, Fablabs, Coworking Spaces, Open Design City, Repair Café, Bikekitchen, Tech Shops, Siebdruckwerkstätten, Hackerspaces oder einfach nur Bastelbuden übernehmen die Funktion offener Ressourcenzentren. Dort kann erprobt, repariert, gedruckt, gelötet oder geschreinert werden. Es bleibt die Frage, ob dies modischer Hype, Lifestyle-Trend oder doch ein Aufbruch in eine nachhaltige Wirtschaft ist?

\section{Die Praxis des Selber- machens}

Die Praxis der Maker, so meine These, zielt nicht ausschließlich auf Produktionsautonomie, sondern vielmehr auf die Wiedererlangung von communitybasierter Zeitautonomie. Dieses Moment von Hier und Jetzt, von Anti-Utopie und Anti-Prokrastination, von Mach- barkeitsbehauptung der Maker ist als Diskursphänomen bekannt (Baier/Müller/Werner 2013), einerseits in prototypischer Gestalt des produzierenden Bastlers (Anderson 2013), als Techniknerd (Anderegg, 2007) sowie als stigmatisierter Hipster von neuen Trends im Städtischen.

Ein Teil dieser Diskussion fragt momentan, inwiefern es sich bei den Makerkulturen um den im Städtischen sichtbaren Beleg dafür handelt, dass vorindustrielle Produktionsstrukturen in die Stadt zurückkehren und sich im Städtischen breit machen. Reclaim Production ist die logische Folge dessen, was sich mit den Reclaim-Space- und Reclaimthe-City-Bewegungen seit vier bis fünf Jahren weltweit vollzieht.

Derartige neue kleinteilige Produktionsstrukturen finden in der Stadt ihr passendes soziales Umfeld, ebenso wie städtische Dichte diese Produktion erst möglich macht. Die Prototypen dieser Maker-Prozesse werden als gute Menschen in Peergroups und Social Media gewürdigt, idealisiert und auch ein wenig überzeichnet. Wenig beachtet ist hingegen der Zusammenhang zwischen der Dimension Zeit und den damit verbundenen Praxis- und Sozialitätsformen der Maker.

Zwei Varianten geben sich dabei zu erkennen. Zum einen der Prototyp des dynamischen und hyperaktiven Suchers und Finders, bei dem sich die Praxis des Makers mit einem hohen Maß an Handlungsgeschwindigkeit auszeichnet. Schnell, sicher und zielstrebig scheint er, aufgrund seiner technischen Fähigkeit, in der Lage zu sein, sich die Baupläne großer technologischer Zusammenhänge in Do-it-yourself-Manier für sich und seine Community zu eigen zu machen. Unabhängig und schnell, getrieben von der Neugier, die Schalt- und Steuerpläne von elektronischen Alltagsgegenständen wie Fernbedienung, Fön oder Fritteuse bis zu komplexen Techniken wie Drohnen oder Maschenpistolen $\mathrm{zu}$ hacken und neu in Wert zu setzen, avanciert der Maker in jüngster Zeit zum heroischen Sinnbild einer smarten Sozialfigur im Urbanen. Die dabei zum Ausdruck kommenden innovativen Praxisvollzüge machen große Unternehmen und Technikkonzerne extrem neugierig. Im Fokus des Interesses steht zum einen die Frage, inwiefern die Ergebnisse und Produkte der Maker die etablierte Wissens- und Forschungsstrukturen informieren können. Zum anderen streckt zum Beispiel Red Bull seit ein bis zwei Jahren seine Fühler in die wachsende Makerszene aus und unterstützt aktuell den Bau mobiler Labs auf Lastwagen, in denen Maker zu neuen Höhenflügen ansetzen und direkt zu Schülern auf den Schulhof kommen.

Beide Fragen und Prozesse weisen auf die hohen Erwartungen hin, derartige Produktionskontexte in den etablierten Wirtschaftskreisläufen zu integrieren und zu verwerten. Meiner Meinung nach wird zu wenig Augenmerk auf die neu entworfenen sozialen Architekturen der Maker jenseits ihrer Produkte gelegt. Zum einen, weil bis dato Maker meistens als Experten für schnelles, avantgardistisches Re-Kombinieren und redesignen von technologischen Artefakten und deren Systemen gesehen wurden. Zum anderen, weil darin eine Option für marketingfähige, konsumtaugliche Early Adopters und Lead User vermutet wurde, die dem Phänomen des Makers nur in Teilen gerecht wird.

Bisher ungeachtet schält sich gerade im Low-Tech-Feld der Graswurzel-Communities eine Szene von Makern heraus, die so gar nicht ins Klischeebild des schnellen, dynamischen, technologiefixierten Produzenten passt. Mit 
dem Fokus auf traditionellen Materialien, konzentrieren sich diese Akteure auf die Exploration und die Wiederverwertung bereits vorhandener Produkte wie Upcycling und Re-Use. Dabei steht eine zentrale Suchbewegung nach der Aneignungsfähigkeit von physischen Materialen im Vordergrund, um eine Wiedereingliederung in alltägliche realweltliche Zusammenhänge zu ermöglichen. Die Baupläne von Holz, verklebten Materialien, Stoffen, Metallen und anderen Gewerken sind anders zu dechiffrieren und zu knacken, als dies Schaltkreise und transparente Technologien mit sich bringen. Die Verwendbarkeit von Holz beispielsweise folgt anderen Nutzungs- und Bearbeitungslogiken als digital-technische Entwicklungen und Produkte, ebenso wie das Rekombinieren von vorhandenen Gewerken wie Stoffen oder Müll einen anderen Praxisverlauf nimmt. Insbesondere geht mit der Suche nach transformierenden Anwendungsbereichen dieser analogen Gebrauchsgewerke eine andere Form des Entwicklungsdesigns einher, als dies in den softwaretechnologie-affinen Makerkulturen der Fall ist.

\section{Beispiele für Makerprojekte}

Material Mafia, ein Kollektiv von Frauen, arbeitet mit Restmaterialien und organisiert mit der Kompetenz zu Materialvermittlung aus recycelten Ressourcen Workshops und Ausbildungsprogramme im Upcycling sowie Kurse in Druckgrafik. Der generische Mehrwert dieser Praxis basiert auf einer Art material-archäologischen Auseinandersetzung um die verschütteten Techniken, Kompetenzen und sensualen Relationen zwischen Mensch und Material, zwischen Produktion und Aneignung, zwischen Herkunft und Nutzung, die neu ausgelotet werden.

Diese Form der Maker sind in meiner Wahrnehmung nicht nur Macherinnen per se und ebenso wenig ausgerichtet auf das schnelle Neukombinieren von brachliegenden Ressourcen, in diesem Fall aus der Verwertung gefallenen Werkund Verbundmaterialien. Die Macherin- nen verstehen sich vielmehr als Relais in entkoppelten Systemzusammenhängen, sie agieren in vertikalen Schnittfeldern quer zu den etablierten Verwertungsund Diskursinstanzen.

Sevengardens ist ein 1996 in Essen gegründeter Färbergarten, zuerst aus dem Einzelinteresse eines Künstlers entstanden, der ethisch-koschere Farben für seine Bilder nutzen wollte. Im Kern zielt der Ansatz von sevengardens durch die gefahrlose Färbergartenarbeit darauf ab, Menschen in die Lage zu versetzen, sich das Wissen und die Fähigkeiten anzueignen, die eigene Umwelt zu gestalten und die Kompetenzen in dem daraus resultierendem globalen Netzwerk weiter zu entwickeln.

Neue Produkte entstehen, sie werden fair gehandelt oder regional verarbeitet in Form von Kosmetik, Lacken, Wandfarben, Schminke, Druckfarben, Pflanzensaat. Zudem werden Färberwerkstätten mit Werkzeugen, Textilien und Möbeln ausgestattet. Kurzum, aus der Idee, aus Pflanzen Farbe zu gewinnen, entspannt sich seit mehr als 15 Jahren ein Wertschöpfungsnetzwerk, das nicht an den Grenzen des Ruhrgebiets endet.

Transversal wirken diese Spezialisierungen wiederum in die Bestrebungen des Ruhrgebiets, den Fokus auf Bildung, Klimaschutz und die weitere wirtschaftliche Ausrichtung des bisherigen Projekts zu legen. Sevengardens ist zu dem in 28 Ländern tätigen Projektverbund seven European gardens avanciert und baut Organisationen mit Partnern vor Ort auf.

\section{Fazit}

Ein Teil der neuen Maker-Communities weisen auf eine andere Form der Herstellung von Rationalität hin. In diesen Praxis- und Materialexplorationen wird Zeit und Prozessualität der Gestaltund Bearbeitbarkeit von Materialien gewidmet und diese immer auch an eine sinnlich-körperliche Dimension der Erfahrbarkeit geknüpft. Die Suche nach neuen Verwendungszusammenhängen ist aber nicht gegen Effizienz- und Optimierungsbestrebungen, sondern gerade für Nachhaltigkeit. Wirtschaftlich lassen sich sozial eingebettete Praxisformen nur schwer unternehmerisch skalieren und transferieren. Ortsbindung und geteilte Kultur- und Kommunikationstechniken lassen sich nicht im Sinne eines skalierbaren Wirtschaftsmodells voraussetzungslos vertreiben. Communities müssen selbst immer wieder neue Räume erobern, systemische Kopplungen ausloten und neue Verfahren der Vergemeinschaftung herstellen. Die dabei erworbene soziale Währung der Maker drückt sich in einem Zugewinn an Netzwerkkompetenz, Gestaltungsautonomie und Verfügbarkeit von Zeit aus. Am Beispiel der Maker zeigt sich ein anderes Verständnis von Ertrag und Ertragserwartung. Während die heroischen Makernerds zweifelsohne in Do-it-yourself-Mentalität Ideen in Businessformate überführen, zeigt sich hier eine Umkehrung. Im Sinne eines postheroischen NetzwerkManagements wird mittels Entschleunigung der Produktionsprozesse Gestaltungsautonomie in der Lebensführung zurückgewonnen. Die Neuordnung von Artefakten zu gefügigen Produktionsprozessen bietet den Machern die Möglichkeit, parallel zum Tun und Machen eine zeitlich gesprochen gedehnte Gegenwart zu erfahren. Derartige Gewerke und Materialien fordern wiederum ihre Zeit ein.

\section{Literatur}

Anderegg, David (2007): Nerds. Who They Are and Why We Need More of Them. Penguin.

Anderson, Chris (2013): Makers. Das Internet der Dinge: die nächste industrielle Revolution. München, Hanser.

Baier, Andrea/Müller, Christa/Werner, Karin (2013): Stadt der Commonisten. Neue urbane Räume des Do it yourself. Bielefeld, transcript.

Friebe, Holm/Ramge, Thomas (2008): Marke Eigenbau: der Aufstand der Massen gegen die Massenproduktion. Frankfurt/Main, Campus.

\section{AUTOR + KONTAKT}

Dr. Bastian Lange ist Fellow am Georg-SimmelZentrum für Metropolenforschung an der Humboldt Universität Berlin sowie Geschäftsführer des Strategieberatungsbüros Multiplicities.

Bastian Lange, E-Mail: Lange@multiplicities.de; Internet: www.multiplicities.de 